\title{
Bilateral Luxatio Erecta - Etiology, Diagnosis and Management
}

\section{OPEN ACCESS}

Citation: Bawale R., Soliman A. Jain R., Singh B. (2021) Bilateral Luxatio Erecta- Etiology, Diagnosis and Management. Open Science Journal 6(2)

Received: $14^{\text {th }}$ February 2021

Accepted: $4^{\text {th }}$ March 2021

Published: $5^{\text {th }}$ May 2021

Copyright: (c) 2021 This is an open access article under the terms of the Creative Commons Attribution License, which permits unrestricted use, distribution, and reproduction in any medium, provided the original author and source are credited.

Funding: The author(s) received no specific funding for this work

Competing Interests: The author has declared that no competing interests exists.

\author{
Rajesh Bawale $^{*}$, Amr Soliman ${ }^{1}$, Rohit Jain ${ }^{1}$, Bijayendra Singh ${ }^{1}$ \\ ${ }^{1}$ Medway Maritime Hospital, United Kingdom \\ *Corresponding author: Rajesh Bawale: rajgo135@gmail.com
}

Keywords: Luxatio Erecta, Etiology, Diagnosis.

\section{Introduction}

Inferior dislocation of the glenohumeral joint, also known as luxatio erecta humeri, rarely presents in the emergency department [1]. Its clinical manifestation is characterized by an abducted position of the affected arm, flexed elbow, and pronated forearm. Bilateral luxatio erecta shows a rare presentation, with just a few cases reported in literature with incidence of $0.5 \%$ of all the shoulder dislocations. It is associated with a high incidence of complications, such as neurovascular injury; injuries to the humeral head and glenoid, rotator cuff, clavicle, caspules and ligaments. Hence, it needs prompt diagnosis and management [2]. We report a case of traumatic bilateral luxatio erecta treated without any complications.

\section{Case Report}

A 50-year-old male, builder by profession, fell off the ladder while doing work, and sustained closed bilateral luxatio erecta. The anamnestic research revealed that the patient fell downstairs, used his both arms to hold on to the ladder and while clinging to the ladder steps, patient sustained bilateral shoulder dislocations. Examination showed both shoulders were abducted and flexed at 110-120 degrees, with the elbow partially flexed (Image 1). 


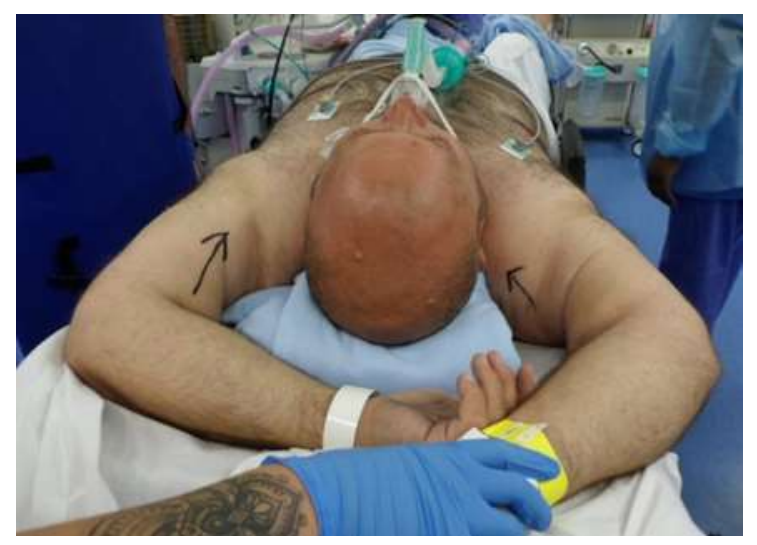

The patient was unable to tolerate active and passive movement of the shoulders due to pain. Both humeral heads were visible and palpable in the lower part of both axillae. No neurovascular deficit was noted for both upper extremities. The patient was brought in as a trauma call due to mechanism of injury hence the trauma CT series was carried out. The CT scan images revealed bilateral luxatio erecta (Images 2,3 and 4) with no associated fractures.

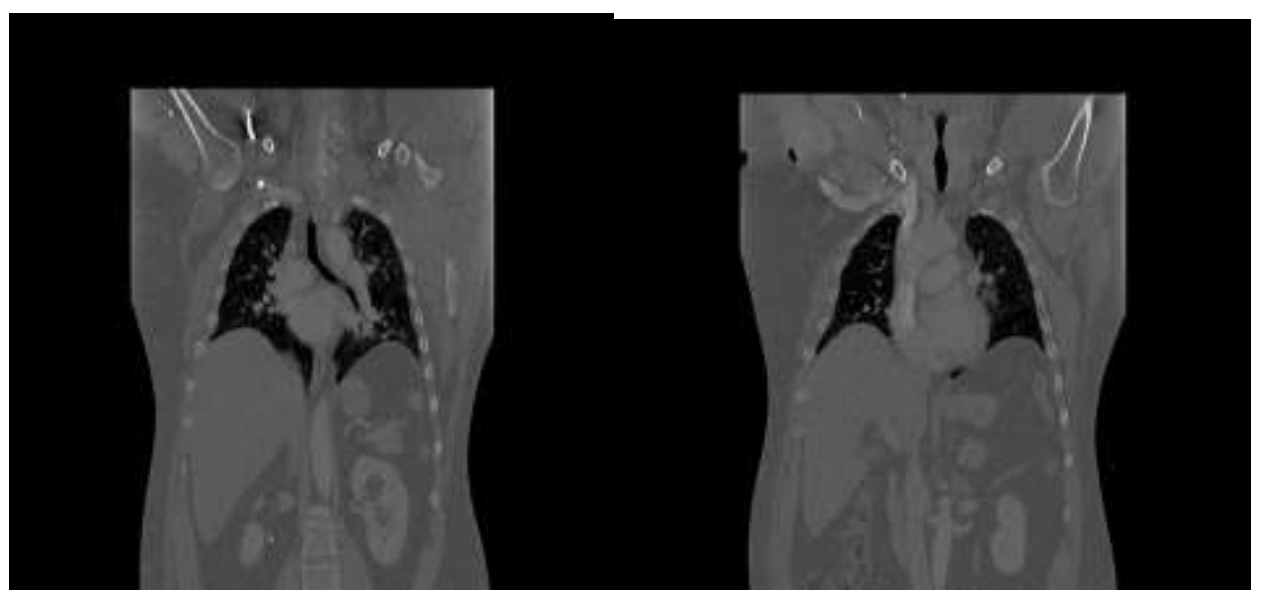

Image 2.Right Luxatio Erecta

Image 3.Left Luxatio Erecta

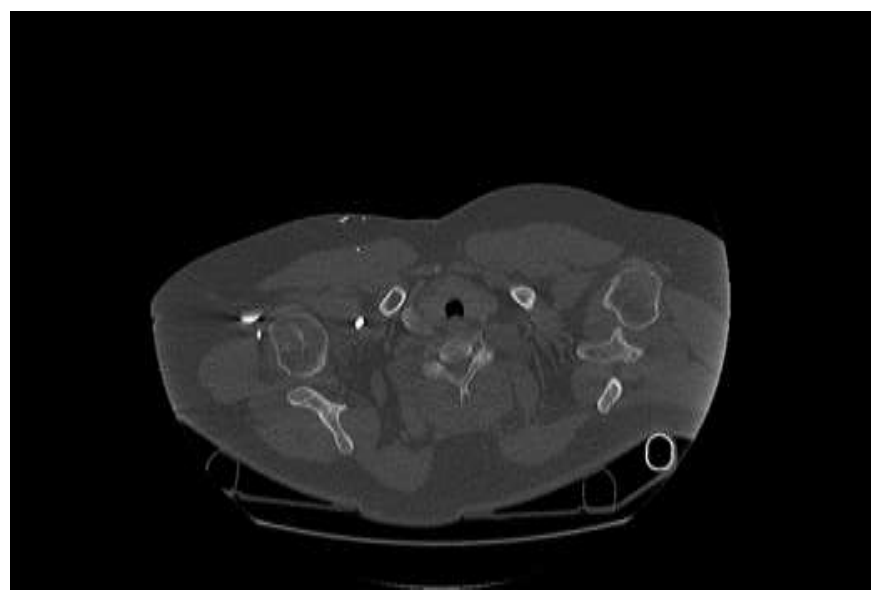

Image 4. Axial section showing bilateral shoulder dislocation

Closed reduction under anaesthesia was performed by applying superior force to the humeral heads, with the axial traction. The image intensifier confirmed the satisfactory anatomical reduction of both humeral heads. Arm slings were then 
applied to both arms and were immobilised in adduction and internal rotation. The clinical examination of both shoulder joints showed normal appearance without the visible and prominent humeral heads (Image 4).

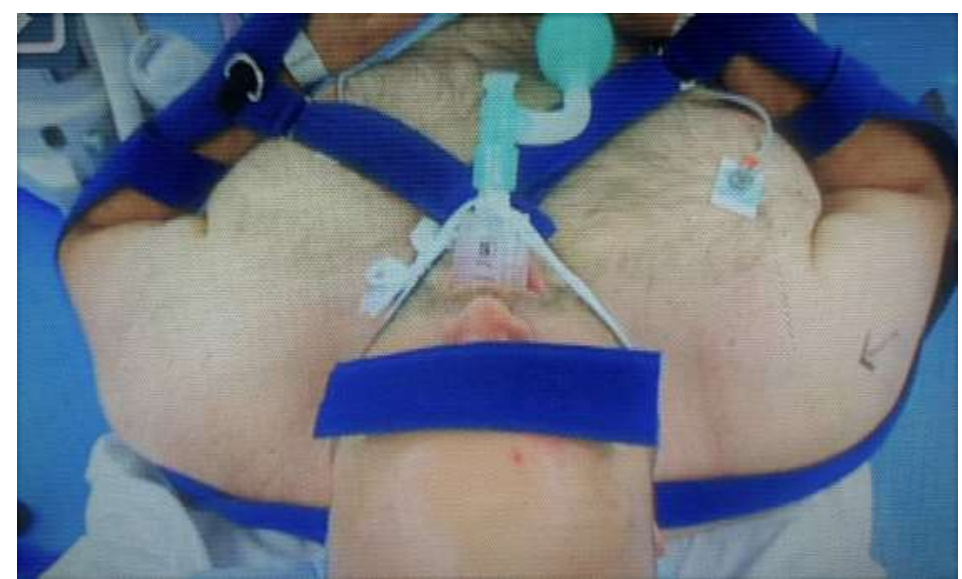

Image 4. Post reduction

The neurovascular status was reassessed and no abnormality was noted. CT scan was repeated, which confirmed anatomical reduction of both shoulder joints without any fracture (Images 5 and 6).

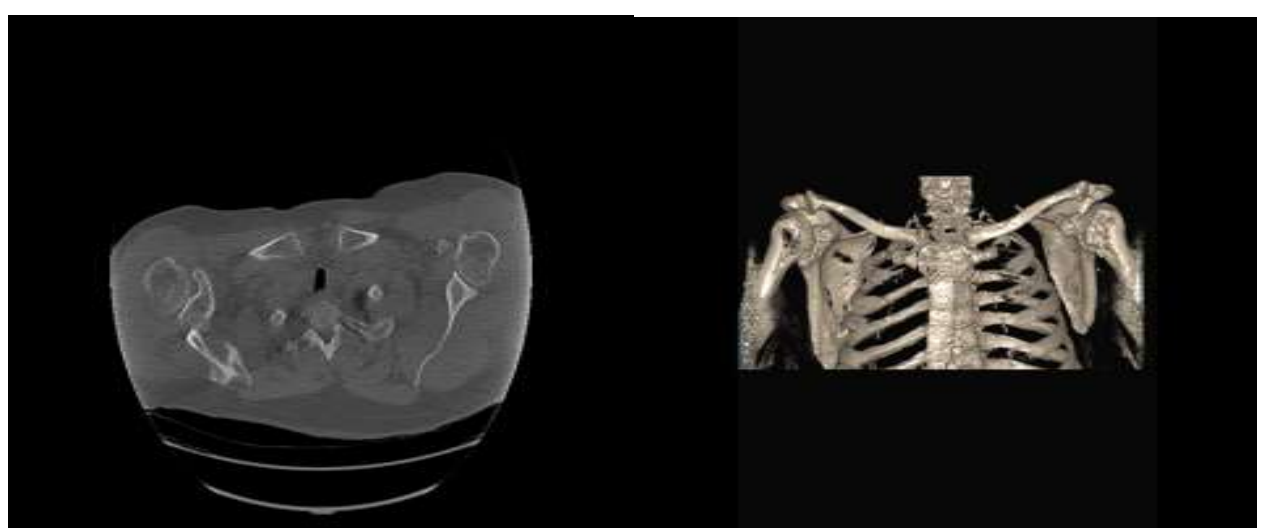

Image 4. Axial section of CT scan

Image 5. 3D CT view

The patient remained stable and was subsequently discharged the next day. The repeat clinical examination revealed altered sensation in the left deltoid region indicating axillary nerve neuropraxia. Progressive and passive mobilisation of the shoulders including pendulum exercises were started after 2 weeks. At 4 weeks post injury, active movement was commenced aiming to achieve a satisfactory range of motion. The patient signed the informed consent.

\section{Discussion}

Dr Middeldorpf and Dr Scharm described the inferior shoulder dislocation in 1859 [3]. Inferior dislocations are rare (0.5\%) and bilateral inferior shoulder dislocations are rare as very few cases have been described in the literature [4]. The mechanism of injury is a violent abduction force of the proximal humerus shaft against the acromion [5]. Direct axial loading from the superior direction is a less common mechanism leading to inferior shoulder dislocation. In this case 
report, the first mechanism was involved. While the patient was sliding down the ladder, his hands were holding onto the steps of the ladder and the arms were abducted. The clinical presentation of the inferior shoulder dislocation is typical, the extremity is held over the head in fixed abducted position and the humeral head is palpable on the chest wall [6]. The single step reduction manoeuvre is traction-counter traction under sedation / general anaesthesia followed by immobilisation for 1-2 weeks. NHo et al described two staged reduction method, which includes transforming the inferior dislocation to an anterior dislocation to reduce the joint in a stable position before the ultimate shoulder joint reduction [7]. The author stated that two stage reduction technique was easier than single stage traction-countertraction technique. The inferior shoulder dislocation is associated with rotator cuff tear (approx. 12\%) and SLAP lesion as seen in arthroscopy procedure [8]. The other less common complications are fracture of acromion, clavicle, inferior glenoid fossa and greater tuberosity. The neurovascular injury is associated with inferior shoulder dislocation [9]. The Axillary nerve is the most common injury followed by brachial plexopathy. The nerve injuries are neuropraxia and recovers well post reduction. The vascular injury involves the axillary vein thrombosis and axillary artery injury are less common than nerve injuries and resolve post reduction with good prognosis and rarely need any major intervention.

\section{Conclusion}

Luxatio erecta can result from a loading force on a fully abducted arm, as well as can be observed in high energy trauma. Single step reduction with adequate anaesthesia/ sedation can be achieved satisfactorily and it's as easy as two step reduction technique.

\section{References}

1.T, Yamamoto, S. Yoshiya, M Kurosaka, K. Nagira, and Y. Nabeshima, "Luxatio erecta (inferior dislocation of the shoulder): a report of 5 cases and a review of the literature,"The American Journal of Orthpaedics, vol.32, no.12, pp. 601-602,2003

2.K. Gokus, E. Sagtas, M Saylik, A. Aydin, and H. Atmaca, "Luxatio erecta humeri: report of a swimming injury with analysis of the mechanism of the injury and associated injuries in literature," Journal of Emergencies, Trauma and Shock, vol.8, no.1, pp.43-48,2015

3.Kumar K.S., O'Rourke S., Pillay J.G. Hands up: a case of bilateral inferior shoulder dislocation. Emergency Medicine Journal. 2001;18(5): 404-405.doi:10.1136/emj.18.5.404.

4.Laskin RS, Sedlin ED (1971). Luxatio Erecta in infancy. Clin Orthop 80:126-129.

5.Rockwood CA, Green DP. Fractures in adult, 1984. Vol 1, Lipincott, Philadelphia, pp 856-860.

6.Freundlich BD (1983) Luxatio erecta. J Trauma 23(5):434-436.

7.S.J. Nho, C.C. Dodson, K.E. Bardzik, R.H. Brophy, B.G. Domb, and J.D. Macgillivray, "The twostep maneuver for closed reduction of inferior glenohumeral dislocation (luxation erecta to anterior shoulder dislocation to reduction)," Journal of Orthopaedic Trauma, Vol. 20, no 5, pp. 334-357, 2006.

8.Schai P, Hintermann B (1998) Arthroscopic findings in luxatio erecta of the glenohumeral joint: case report and review of the literature. Clin J Sport Med 8(2):128-141.

9.Lev-El A, Adar R, Rubinstein Z (1981). Axillary artery injury in erect dislocation of the shoulder. J Trauma 21 (4): 323-325. 